\title{
A more accurate half-discrete Hilbert-type inequality in the whole plane and the reverses
}

\section{Michael Th. Rassias ${ }^{1,2,3} \cdot$ Bicheng Yang $^{4} \cdot$ Gerasimos C. Meletiou $^{5,6}$}

Received: 3 May 2021 / Accepted: 25 May 2021 / Published online: 29 June 2021

(C) The Author(s) 2021

\begin{abstract}
A more accurate half-discrete Hilbert-type inequality in the whole plane with multiparameters is established by the use of Hermite-Hadamard's inequality and weight functions. Furthermore, some equivalent forms and some special types of inequalities and operator representations as well as reverses are considered.
\end{abstract}

Keywords Hermite-Hadamard inequality · Half-discrete Hilbert-type inequality · Weight function $\cdot$ Equivalent form $\cdot$ Operator expression $\cdot$ Reverse

Mathematics Subject Classification 26D15 · 47A05

\section{Introduction}

For $p>1, \frac{1}{p}+\frac{1}{q}=1, a_{m}, b_{n}>0$,

\section{Communicated by M. S. Moslehian.}

Michael Th. Rassias

mthrassias@yahoo.com; michail.rassias@math.uzh.ch

Bicheng Yang

bcyang@gdei.edu.cn; bcyang818@163.com

Gerasimos C. Meletiou

gmelet@uoi.gr; gmelet@neptune.math.upatras.gr

1 Institute of Mathematics University of Zurich, 8057 Zurich, Switzerland

2 Moscow Institute of Physics and Technology, Institutskiy per, d. 9, 141700 Dolgoprudny, Russia

3 Institute for Advanced Study, Program in Interdisciplinary Studies, 1 Einstein Dr, Princeton, NJ 08540, USA

4 Department of Mathematics, Guangdong University of Education, Guangzhou 510303, Guangdong, People's Republic of China

5 Department of Agriculture, University of Ioannina, Arta, Greece

6 Computational Intelligence Laboratory (CILab), University of Patras, Patras, Greece 


$$
0<\sum_{m=1}^{\infty} a_{m}^{p}<\infty \text { and } 0<\sum_{n=1}^{\infty} b_{n}^{q}<\infty,
$$

the following discrete Hardy-Hilbert inequality (cf. [10], Theorem 315, and [4, 11, 36, 40]) holds true:

$$
\sum_{n=1}^{\infty} \sum_{m=1}^{\infty} \frac{a_{m} b_{n}}{m+n}<\frac{\pi}{\sin (\pi / p)}\left(\sum_{m=1}^{\infty} a_{m}^{p}\right)^{\frac{1}{p}}\left(\sum_{n=1}^{\infty} b_{n}^{q}\right)^{\frac{1}{q}} .
$$

The constant factor $\frac{\pi}{\sin (\pi / p)}$ is optimal.

Let $f(x), g(y) \geq 0$, such that

$$
0<\int_{0}^{\infty} f^{p}(x) \mathrm{d} x<\infty \text { and } 0<\int_{0}^{\infty} g^{q}(y) d y<\infty .
$$

Then the following Hardy-Hilbert integral inequality with the same best possible constant factor $\frac{\pi}{\sin (\pi / p)}$ (cf. [11], Theorem 316) is valid:

$$
\int_{0}^{\infty} \int_{0}^{\infty} \frac{f(x) g(y)}{x+y} \mathrm{~d} x d y<\frac{\pi}{\sin (\pi / p)}\left(\int_{0}^{\infty} f^{p}(x) \mathrm{d} x\right)^{\frac{1}{p}}\left(\int_{0}^{\infty} g^{q}(y) d y\right)^{\frac{1}{q}} .
$$

The following half-discrete Hardy-Hilbert inequality with the same best possible constant factor was recently formulated and proved (cf. [39]):

$$
\sum_{n=1}^{\infty} \int_{0}^{\infty} \frac{b_{n} f(x)}{x+n} \mathrm{~d} x<\frac{\pi}{\sin (\pi / p)}\left(\int_{0}^{\infty} f^{p}(x) \mathrm{d} x\right)^{\frac{1}{p}}\left(\sum_{n=1}^{\infty} b_{n}^{q}\right)^{\frac{1}{q}} .
$$

Several inequalities with homogenous kernels of degree 0 as well as with nonhomogenous kernels have been proved in [7, 11, 19, 37, 42, 45]. For a large variety of integral inequalities of Hilbert-type the interested reader is referred to $[1-6,8$, $12,15,16,20,22-24,26,41,46,47]$. The above inequalities are constructed in the quarter plane of the first quadrant.

A Hilbert-type integral inequality in the whole plane was proved in [33] by Yang. Furthermore, a generalized form of a Hilbert-type integral inequality in the whole plane was considered in [34]:

$$
\begin{aligned}
& \int_{-\infty}^{\infty} \int_{-\infty}^{\infty} \frac{1}{|1+x y|^{\lambda}} f(x) g(y) \mathrm{d} x d y \\
& \quad<k_{\lambda}\left[\int_{-\infty}^{\infty}|x|^{p\left(1-\frac{\lambda}{2}\right)-1} f^{p}(x) \mathrm{d} x\right]^{\frac{1}{p}}\left[\int_{-\infty}^{\infty}|y|^{q\left(1-\frac{\lambda}{2}\right)-1} g^{q}(y) d y\right]^{\frac{1}{q}} .
\end{aligned}
$$

The constant factor

$$
k_{\lambda}=B\left(\frac{\lambda}{2}, \frac{\lambda}{2}\right)+2 B\left(1-\lambda, \frac{\lambda}{2}\right)(0<\lambda<1)
$$


is optimal. Additionally, in [9, 13, 14, 25, 27, 29, 30, 43, 44] several integral and discrete Hilbert-type inequalities in the whole plane where formulated and proved.

The goal of the present paper is to study the following half-discrete Hilbert-type inequality in the whole plane with parameters and a best possible constant factor, by applying the Hermite-Hadamard inequality and weight functions:

$$
\begin{aligned}
& \sum_{|n|=1}^{\infty} \int_{-\infty}^{\infty} \frac{\ln \left(\frac{|n|}{|x|}\right)}{|n|^{\lambda}-|x|^{\lambda}} f(x) b_{n} \mathrm{~d} x<2\left[\frac{\pi}{\lambda \sin \left(\frac{\pi \sigma}{\lambda}\right)}\right]^{2} \\
& \times\left[\int_{-\infty}^{\infty}|x|^{p(1-\mu)-1} f^{p}(x) \mathrm{d} x\right]^{\frac{1}{p}}\left[\sum_{|n|=1}^{\infty}|n|^{q(1-\sigma)-1} b_{n}^{q}\right]^{\frac{1}{q}},
\end{aligned}
$$

where $\sigma, \mu>0, \sigma+\mu=\lambda \leq 1$. Moreover, a more accurate half-discrete Hilbert-type inequality with multiparameters is proved. Some equivalent forms, a few special types of inequalities as well as operator representations and reverses are studied.

\section{Some lemmas}

In what follows, we assume that $\delta \in\{-1,1\}, a, b \in(-1,1), \sigma, \mu>0, \sigma+\mu=\lambda \leq 1$, $\xi \in(-\infty, \infty), \eta \in\left[0, \frac{1}{2}\right]$. We set

$$
H(x, y):=\frac{\ln \left(\frac{|y-\eta|+b(y-\eta)}{[|x-\xi|+a(x-\xi)]^{\delta}}\right)}{\left(\frac{|y-\eta|+b(y-\eta)}{[|x-\xi|+a(x-\xi)]^{\delta}}\right)^{\lambda}-1}(x \neq \xi, y \neq \eta),
$$

wherefrom

$$
\begin{aligned}
H(x, y) & =\frac{\ln \left(\frac{(y-\eta)(1+b)}{[|x-\xi|+a(x-\xi)]^{\delta}}\right)}{\left(\frac{|y-\eta|+b(y-\eta)}{[|x-\xi|+a(x-\xi)]^{\delta}}\right)^{\lambda}-1}(y>\eta), \\
H(x, y) & =\frac{\ln \left(\frac{|y-\eta|+b(y-\eta)}{[(x-\xi)(1+a)]^{\delta}}\right)}{\left(\frac{|y-\eta|+b(y-\eta)}{[(x-\xi)(1+a)]^{\delta}}\right)^{\lambda}-1}(x>\xi), \\
H(-x, y) & =\frac{\ln \left(\frac{|y-\eta|+b(y-\eta)}{[(x+\xi)(1-a)]^{\delta}}\right)}{\left(\frac{|y-\eta|+b(y-\eta)}{[(x+\xi)(1-a)]^{\delta}}\right)^{\lambda}-1}(x>-\xi), \\
H(x,-y) & =\frac{\left(\frac{(y+\eta)(1-b)}{[|x-\xi|+a(x-\xi)]^{\delta}}\right)}{\left(\frac{(y+\eta)(1-b)}{[|x-\xi|+a(x-\xi)]^{\delta}}\right)^{\lambda}-1}(y>-\eta) .
\end{aligned}
$$


Lemma 2.1 We define two weight functions $\omega(\sigma, n)$ and $\varpi(\sigma, x)$ as follows:

$$
\begin{gathered}
\omega(\sigma, n):=\int_{-\infty}^{\infty} H(x, n) \frac{[|n-\eta|+b(n-\eta)]^{\sigma} \mathrm{d} x}{[|x-\xi|+a(x-\xi)]^{1+\delta \sigma}}(|n| \in \mathbf{N}), \\
\varpi(\sigma, x):=\sum_{|n|=1}^{\infty} H(x, n) \frac{[|x-\xi|+a(x-\xi)]^{-\delta \sigma}}{[|n-\eta|+b(n-\eta)]^{1-\sigma}}(x \in \mathbf{R} \backslash\{\xi\}) .
\end{gathered}
$$

Then:

(i) we have

$$
\omega(\sigma, n)=k_{a}(\sigma):=\frac{2}{1-a^{2}}\left[\frac{\pi}{\lambda \sin \left(\frac{\pi \sigma}{\lambda}\right)}\right]^{2} \in \mathbf{R}_{+}(|n| \in \mathbf{N}) ;
$$

(ii) we also have

$$
k_{b}(\sigma)(1-\theta(\sigma, x))<\varpi(\sigma, x)<k_{b}(\sigma)(x \in \mathbf{R} \backslash\{\xi\}),
$$

where

$$
k_{b}(\sigma)=\frac{2}{1-b^{2}}\left[\frac{\pi}{\lambda \sin \left(\frac{\pi \sigma}{\lambda}\right)}\right]^{2}
$$

and

$$
\begin{aligned}
\theta(\sigma, x) & :=\left[\frac{\sin \left(\frac{\pi \sigma}{\lambda}\right)}{\pi}\right]_{0}^{2} \int_{0}^{\left\{\frac{(1+\eta)(1+b)}{[|x-\xi|+a(x-\xi)]^{\delta}}\right\}^{\lambda}} \frac{\ln u}{u-1} u^{\frac{\sigma}{\lambda}-1} \mathrm{~d} u \\
& =O\left(\frac{1}{[|x-\xi|+a(x-\xi)]^{\delta(\sigma-\lambda \kappa)}}\right) \in(0,1)\left(0<\kappa<\frac{\sigma}{\lambda}\right) .
\end{aligned}
$$

Proof It holds that 


$$
\begin{aligned}
\omega(\sigma, n)= & \int_{-\infty}^{\xi} H(x, n) \frac{[|n-\eta|+b(n-\eta)]^{\sigma}}{[(x-\xi)(a-1)]^{1+\delta \sigma}} \mathrm{d} x \\
& +\int_{\xi}^{\infty} H(x, n) \frac{[|n-\eta|+b(n-\eta)]^{\sigma}}{[(x-\xi)(a+1)]^{1+\delta \sigma}} \mathrm{d} x \\
= & \int_{-\xi}^{\infty} H(-x, n) \frac{[|n-\eta|+b(n-\eta)]^{\sigma}}{[(x+\xi)(1-a)]^{1+\delta \sigma}} \mathrm{d} x \\
& +\int_{\xi}^{\infty} H(x, n) \frac{[|n-\eta|+b(n-\eta)]^{\sigma}}{[(x-\xi)(1+a)]^{1+\delta \sigma}} \mathrm{d} x .
\end{aligned}
$$

Setting

$$
u=\left(\frac{[|n-\eta|+b(n-\eta)]}{[(x+\xi)(1-a)]^{\delta}}\right)^{\lambda}\left(\text { resp. } u=\left(\frac{[|n-\eta|+b(n-\eta)]}{[(x-\xi)(1+a)]^{\delta}}\right)^{\lambda}\right)
$$

in the above first (resp. second) integral, by simplifications, we deduce that

$$
\begin{aligned}
\omega(\sigma, n)= & \frac{1}{\lambda^{2}(1-a)} \int_{0}^{\infty} \frac{\ln u}{u-1} u^{\frac{\sigma}{\lambda}-1} \mathrm{~d} u \\
& +\frac{1}{\lambda^{2}(1+a)} \int_{0}^{\infty} \frac{\ln u}{u-1} u^{\frac{\sigma}{\lambda}-1} \mathrm{~d} u \\
= & \frac{2}{\lambda^{2}\left(1-a^{2}\right)}\left[\frac{\pi}{\sin \left(\frac{\pi \sigma}{\lambda}\right)}\right]^{2} .
\end{aligned}
$$

Hence, (8) follows.

We obtain

$$
\begin{aligned}
\varpi(\sigma, x)= & \sum_{n=-1}^{-\infty} H(x, n) \frac{[|x-\xi|+a(x-\xi)]^{-\delta \sigma}}{[|n-\eta|+b(n-\eta)]^{1-\sigma}} \\
& +\sum_{n=1}^{\infty} H(x, n) \frac{[|x-\xi|+a(x-\xi)]^{-\delta \sigma}}{[|n-\eta|+b(n-\eta)]^{1-\sigma}} \\
= & \frac{[|x-\xi|+a(x-\xi)]^{-\delta \sigma}}{(1-b)^{1-\sigma}} \sum_{n=1}^{\infty} \frac{H(x,-n)}{(n+\eta)^{1-\sigma}} \\
& +\frac{[|x-\xi|+a(x-\xi)]^{-\delta \sigma}}{(1+b)^{1-\sigma}} \sum_{n=1}^{\infty} \frac{H(x, n)}{(n-\eta)^{1-\sigma}} .
\end{aligned}
$$

For $0<\sigma<\lambda \leq 1$, since $(-1)^{i} \frac{d^{(i)}}{\mathrm{d} u^{(i)}} \frac{\ln u}{u^{\lambda}-1}>0(i=1,2)$ (cf. [36]), we find that both $\frac{H(x,-y)}{(y+\eta)^{1-\sigma}}$ and $\frac{H(x, y)}{(y-\eta)^{1-\sigma}}$ are strictly decreasing and strictly convex in $y \in\left(\frac{1}{2}, \infty\right)$, satisfying 


$$
(-1)^{i} \frac{d^{(i)}}{d y^{(i)}} \frac{H(x,-y)}{(y+\eta)^{1-\sigma}}>0,(-1)^{i} \frac{d^{(i)}}{d y^{(i)}} \frac{H(x, y)}{(y-\eta)^{1-\sigma}}>0(i=1,2) .
$$

By (11) and Hermite-Hadamard's inequality (cf. [17]), in view of $\eta \in\left[0, \frac{1}{2}\right]$, we have that

$$
\begin{aligned}
\varpi(\sigma, x)< & \frac{[|x-\xi|+a(x-\xi)]^{-\delta \sigma}}{(1-b)^{1-\sigma}} \int_{\frac{1}{2}}^{\infty} \frac{H(x,-y)}{(y+\eta)^{1-\sigma}} d y \\
& +\frac{[|x-\xi|+a(x-\xi)]^{-\delta \sigma}}{(1+b)^{1-\sigma}} \int_{\frac{1}{2}}^{\infty} \frac{H(x, y)}{(y-\eta)^{1-\sigma}} d y \\
\leq & \frac{[|x-\xi|+a(x-\xi)]^{-\delta \sigma}}{(1-b)^{1-\sigma}} \int_{-\eta}^{\infty} \frac{H(x,-y) d y}{(y+\eta)^{1-\sigma}} \\
& +\frac{[|x-\xi|+a(x-\xi)]^{-\delta \sigma}}{(1+b)^{1-\sigma}} \int_{\eta}^{\infty} \frac{H(x, y) d y}{(y-\eta)^{1-\sigma}}
\end{aligned}
$$

Setting

$$
u=\left\{\frac{(y+\eta)(1-b)}{[|x-\xi|+a(x-\xi)]^{\delta}}\right\}^{\lambda} \quad\left(\text { resp. } u=\left\{\frac{(y-\eta)(1+b)}{[|x-\xi|+a(x-\xi)]^{\delta}}\right\}^{\lambda}\right)
$$

in the above first (resp. second) integral, by simplifications, we deduce that

$$
\varpi(\sigma, x)<\frac{1}{1-b^{2}}\left[\frac{\pi}{\lambda \sin \left(\frac{\pi \sigma}{\lambda}\right)}\right]^{2}=k_{b}(\sigma) .
$$

By (11) and the decreasing property of series, we also have that

$$
\begin{aligned}
\varpi(\sigma, x)> & \frac{[|x-\xi|+a(x-\xi)]^{-\delta \sigma}}{(1-b)^{1-\sigma}} \int_{1}^{\infty} \frac{H(x,-y)}{(y+\eta)^{1-\sigma}} d y \\
& +\frac{[|x-\xi|+a(x-\xi)]^{-\delta \sigma}}{(1+b)^{1-\sigma}} \int_{1}^{\infty} \frac{H(x, y)}{(y-\eta)^{1-\sigma}} d y .
\end{aligned}
$$

Setting

$$
u=\left\{\frac{(y+\eta)(1-b)}{[|x-\xi|+a(x-\xi)]^{\delta}}\right\}^{\lambda} \quad\left(\text { resp. } u=\left\{\frac{(y-\eta)(1+b)}{[|x-\xi|+a(x-\xi)]^{\delta}}\right\}^{\lambda}\right)
$$

in the above first (resp. second) integral, by simplifications, we derive that 


$$
\begin{aligned}
\varpi(\sigma, x)> & \frac{1}{\lambda^{2}(1-b)} \int_{\left\{\frac{(1+\eta)(1-b)}{[|x-\xi|+a(x-\xi)]^{\delta}}\right\}^{\lambda}}^{\infty} \frac{\ln u}{u-1} u^{\frac{\sigma}{\lambda}-1} \mathrm{~d} u \\
& +\frac{1}{\lambda^{2}(1+b)} \int_{\left\{\frac{(1-\eta)(1+b)}{[|x-\xi|+a(x-\xi)]^{\delta}}\right.}^{\infty} \frac{\ln u}{u-1} u^{\frac{\sigma}{\lambda}-1} \mathrm{~d} u \\
\geq & \frac{2}{\lambda^{2}\left(1-b^{2}\right)} \int_{\left\{\frac{(1+\eta)(1+b)}{[|x-\xi|+a(x-\xi)]^{\delta}}\right\}^{\lambda}}^{\infty} \frac{\ln u}{u-1} u^{\frac{\sigma}{\lambda}-1} d \mathrm{~d} u \\
= & k_{b}(\sigma)(1-\theta(\sigma, x))>0 .
\end{aligned}
$$

For some $\kappa \in\left(0, \frac{\sigma}{\lambda}\right)$, we obtain that

$$
\lim _{u \rightarrow 0^{+}} u^{\kappa} \frac{\ln u}{u-1}=\lim _{u \rightarrow \infty} u^{\kappa} \frac{\ln u}{u-1}=0
$$

and thus there exists a constant $L>0$, such that $0<u^{\kappa} \frac{\ln u}{u-1} \leq L(u \in(0, \infty))$. Hence, we have

$$
\begin{aligned}
0 & <\theta(\sigma, x)=\left[\frac{\sin \left(\frac{\pi \sigma}{\lambda}\right)}{\pi}\right]^{2} \int_{0}^{\left\{\frac{(1+\eta)(1+b)}{[|x-\xi|+a(x-\xi)]^{\delta}}\right\}^{\lambda}} u^{\frac{\sigma}{\lambda}-\kappa-1}\left(u^{\kappa} \frac{\ln u}{u-1}\right) \mathrm{d} u \\
& \leq\left[\frac{\sin \left(\frac{\pi \sigma}{\lambda}\right)}{\pi}\right]^{2} L \int_{0}^{\left\{\frac{(1+\eta)(1+b)}{[|x-\xi|+a(x-\xi)]^{\delta}}\right\}^{\lambda}} u^{\frac{\sigma}{\lambda}-\kappa-1} \mathrm{~d} u \\
& =\frac{L}{\left(\frac{\sigma}{\lambda}-\kappa\right)}\left[\frac{\sin \left(\frac{\pi \sigma}{\lambda}\right)}{\pi}\right]^{2}\left\{\frac{(1+\eta)(1+b)}{[|x-\xi|+a(x-\xi)]^{\delta}}\right\}^{\sigma-\kappa \lambda},
\end{aligned}
$$

and therefore (9) and (10) follow.

This completes the proof of the lemma.

Lemma 2.2 For $\varepsilon>0$,

$$
H_{\varepsilon}(b):=\sum_{|n|=1}^{\infty} \frac{1}{[|n-\eta|+b(n-\eta)]^{1+\varepsilon}},
$$

we have

$$
H_{\varepsilon}(b)=\frac{1}{\varepsilon}\left(\frac{2}{1-b^{2}}+o_{1}(1)\right)\left(1+o_{2}(1)\right)\left(\varepsilon \rightarrow 0^{+}\right) .
$$

Proof It holds that 


$$
\begin{aligned}
H_{\varepsilon}(b) & =\sum_{n=-1}^{-\infty} \frac{1}{[(n-\eta)(b-1)]^{1+\varepsilon}}+\sum_{n=1}^{\infty} \frac{1}{[(n-\eta)(b+1)]^{1+\varepsilon}} \\
& =\frac{1}{(1-b)^{1+\varepsilon}} \sum_{n=1}^{\infty} \frac{1}{(n+\eta)^{1+\varepsilon}}+\frac{1}{(1+b)^{1+\varepsilon}} \sum_{n=1}^{\infty} \frac{1}{(n-\eta)^{1+\varepsilon}} .
\end{aligned}
$$

By (13) and the decreasing property of series, we derive that

$$
\begin{aligned}
H_{\varepsilon}(b) & \leq\left[\frac{1}{(1+b)^{1+\varepsilon}}+\frac{1}{(1-b)^{1+\varepsilon}}\right] \sum_{n=1}^{\infty} \frac{1}{(n-\eta)^{1+\varepsilon}} \\
& =\left[\frac{1}{(1+b)^{1+\varepsilon}}+\frac{1}{(1-b)^{1+\varepsilon}}\right]\left[\frac{1}{(1-\eta)^{1+\varepsilon}}+\sum_{n=2}^{\infty} \frac{1}{(n-\eta)^{1+\varepsilon}}\right] \\
& <\left[\frac{1}{(1+b)^{1+\varepsilon}}+\frac{1}{(1-b)^{1+\varepsilon}}\right]\left[\frac{1}{(1-\eta)^{1+\varepsilon}}+\int_{1}^{\infty} \frac{d y}{(y-\eta)^{1+\varepsilon}}\right] \\
& =\frac{1}{\varepsilon}\left(\frac{2}{1-b^{2}}+o_{1}(1)\right)\left\{1+\left[\frac{\varepsilon}{(1-\eta)^{1+\varepsilon}}+\frac{1}{(1-\eta)^{\varepsilon}}-1\right]\right\}, \\
H_{\varepsilon}(b) & \geq\left[\frac{1}{(1+b)^{1+\varepsilon}}+\frac{1}{(1-b)^{1+\varepsilon}}\right] \sum_{n=1}^{\infty} \frac{1}{(n+\eta)^{1+\varepsilon}} \\
& >\left[\frac{1}{(1+b)^{1+\varepsilon}}+\frac{1}{(1-b)^{1+\varepsilon}}\right] \int_{1}^{\infty} \frac{d y}{(y+\eta)^{1+\varepsilon}} \\
& =\frac{1}{\varepsilon}\left(\frac{2}{1-b^{2}}+o_{1}(1)\right)\left\{1+\left[\frac{1}{(1+\eta)^{\varepsilon}}-1\right]\right\} .
\end{aligned}
$$

Hence, we obtain (12) and the lemma is proved.

Lemma 2.3 For $\varepsilon>0$, setting

$$
E_{\delta}:=\left\{x \in \mathbf{R} \backslash\{\xi\} ; \frac{1}{[|x-\xi|+a(x-\xi)]^{\delta}} \geq 1\right\},
$$

we have

$$
H_{\delta}:=\int_{E_{\delta}} \frac{1}{[|x-\xi|+a(x-\xi)]^{1+\delta \varepsilon}} \mathrm{d} x=\frac{1}{\varepsilon} \frac{2}{1-a^{2}} .
$$

Proof Setting

$$
\begin{aligned}
E_{\delta}^{+}: & =\left\{x>\xi ; \frac{1}{[(x-\xi)(1+a)]^{\delta}} \geq 1\right\}, \\
E_{\delta}^{-}: & =\left\{x<\xi ; \frac{1}{[(\xi-x)(1-a)]^{\delta}} \geq 1\right\},
\end{aligned}
$$


it follows that $E_{\delta}=E_{\delta}^{+} \cup E_{\delta}^{-}$. We have that

$$
\begin{aligned}
H_{\delta}= & \frac{1}{(1+a)^{1+\delta \varepsilon}} \int_{E_{\delta}^{+}} \frac{1}{(x-\xi)^{1+\delta \varepsilon}} \mathrm{d} x \\
& +\frac{1}{(1-a)^{1+\delta \varepsilon}} \int_{E_{\delta}^{-}} \frac{1}{(\xi-x)^{1+\delta \varepsilon}} \mathrm{d} x .
\end{aligned}
$$

Setting $u=[(x-\xi)(1+a)]^{\delta}$ (resp. $\left.u=[(\xi-x)(1-a)]^{\delta}\right)$ in the above first (resp. second) integral, we obtain

$$
H_{\delta}=\left(\frac{1}{1+a}+\frac{1}{1-a}\right) \int_{1}^{\infty} \frac{\mathrm{d} u}{u^{1+\varepsilon}}=\frac{1}{\varepsilon} \frac{2}{1-a^{2}} .
$$

Hence, we get (14) and thus the lemma is proved.

\section{Main results}

Theorem 3.1 Suppose that $p>1, \frac{1}{p}+\frac{1}{q}=1$,

$$
K_{a, b}(\sigma):=k_{a}^{\frac{1}{q}}(\sigma) k_{b}^{\frac{1}{p}}(\sigma)=\frac{2\left[\frac{\pi}{\lambda \sin \left(\frac{\pi \sigma}{\lambda}\right)}\right]^{2}}{\left(1-a^{2}\right)^{1 / q}\left(1-b^{2}\right)^{1 / p}} .
$$

If $f(x), b_{n} \geq 0$, satisfying

$$
\begin{aligned}
& 0<\int_{-\infty}^{\infty}[|x-\xi|+a(x-\xi)]^{p(1+\delta \sigma)-1} f^{p}(x) \mathrm{d} x<\infty, \text { and } \\
& 0<\sum_{|n|=1}^{\infty}[|n-\eta|+b(n-\eta)]^{q(1-\sigma)-1} b_{n}^{q}<\infty,
\end{aligned}
$$

then we have the following equivalent inequalities:

$$
\begin{aligned}
I:= & \sum_{|n|=1}^{\infty} \int_{-\infty}^{\infty} H(x, n) f(x) b_{n} \mathrm{~d} x \\
< & K_{\alpha, \beta}(\sigma)\left\{\int_{-\infty}^{\infty}[|x-\xi|+a(x-\xi)]^{p(1+\delta \sigma)-1} f^{p}(x) \mathrm{d} x\right\}^{\frac{1}{p}} \\
& \times\left\{\sum_{|n|=1}^{\infty}[|n-\eta|+b(n-\eta)]^{q(1-\sigma)-1} b_{n}^{q}\right\}^{\frac{1}{q}}
\end{aligned}
$$




$$
\begin{aligned}
J_{1}: & =\left\{\sum_{|n|=1}^{\infty}[|n-\eta|+b(n-\eta)]^{p \sigma-1}\left(\int_{-\infty}^{\infty} H(x, n) f(x) \mathrm{d} x\right)^{p}\right\}^{\frac{1}{p}} \\
& <K_{\alpha, \beta}(\sigma)\left\{\int_{-\infty}^{\infty}[|x-\xi|+a(x-\xi)]^{p(1+\delta \sigma)-1} f^{p}(x) \mathrm{d} x\right\}^{\frac{1}{p}}, \\
J_{2}: & =\left\{\int_{-\infty}^{\infty}[|x-\xi|+a(x-\xi)]^{-q \delta \sigma-1}\left(\sum_{|n|=1}^{\infty} H(x, n) b_{n}\right)^{q} \mathrm{~d} x\right\}^{\frac{1}{q}} \\
& <K_{\alpha, \beta}(\sigma)\left\{\sum_{|n|=1}^{\infty}[|n-\eta|+b(n-\eta)]^{q(1-\sigma)-1} b_{n}^{q}\right\}^{\frac{1}{q}} .
\end{aligned}
$$

In particular, for $a=b=0$ we have the following equivalent inequalities:

$$
\begin{aligned}
& \sum_{|n|=1}^{\infty} \int_{-\infty}^{\infty} \frac{\ln \left(\frac{|n-\eta|}{|x-\xi|^{\delta}}\right)}{\left(\frac{|n-\eta|}{|x-\xi|^{\delta}}\right)^{\lambda}-1} f(x) b_{n} \mathrm{~d} x \\
& <\left[\frac{\pi}{\lambda \sin \left(\frac{\pi \sigma}{\lambda}\right)}\right]^{2}\left[\int_{-\infty}^{\infty}|x-\xi|^{p(1+\delta \sigma)-1} f^{p}(x) \mathrm{d} x\right]^{\frac{1}{p}} \\
& \times\left[\sum_{|n|=1}^{\infty}|n-\eta|^{q(1-\sigma)-1} b_{n}^{q}\right]^{\frac{1}{q}}, \\
& \left\{\sum_{|n|=1}^{\infty}|n-\eta|^{p \sigma-1}\left[\int_{-\infty}^{\infty} \frac{\ln \left(\frac{|n-\eta|}{|x-\xi|^{\delta}}\right)}{\left(\frac{|n-\eta|}{|x-\xi|^{\delta}}\right)^{\lambda}-1} f(x) \mathrm{d} x\right]^{p}\right\}^{\frac{1}{p}} \\
& <\left[\frac{\pi}{\lambda \sin \left(\frac{\pi \sigma}{\lambda}\right)}\right]^{2}\left[\int_{-\infty}^{\infty}|x-\xi|^{p(1+\delta \sigma)-1} f^{p}(x) \mathrm{d} x\right]^{\frac{1}{p}}, \\
& \left\{\int_{-\infty}^{\infty}|x-\xi|^{-q \delta \sigma-1}\left[\sum_{|n|=1}^{\infty} \frac{\ln \left(\frac{|n-\eta|}{|x-\xi|^{\delta}}\right)}{\left(\frac{|n-\eta|}{|x-\xi|^{\delta}}\right)^{\lambda}-1} b_{n}\right]^{q} \mathrm{~d} x\right\}^{\frac{1}{q}} \\
& <\left[\frac{\pi}{\lambda \sin \left(\frac{\pi \sigma}{\lambda}\right)}\right]^{2}\left[\sum_{|n|=1}^{\infty}|n-\eta|^{q(1-\sigma)-1} b_{n}^{q}\right]^{\frac{1}{q}} \text {. }
\end{aligned}
$$


Proof By Hölder's inequality (cf. [17]) and (6), we obtain that

$$
\begin{aligned}
& {\left[\int_{-\infty}^{\infty} H(x, n) f(x) \mathrm{d} x\right]^{p} } \\
&=\left\{\int_{-\infty}^{\infty} H(x, n) \frac{[|x-\xi|+a(x-\xi)]^{(1+\delta \sigma) / q}}{[|n-\eta|+b(n-\eta)]^{(1-\sigma) / p}} f(x)\right. \\
&\left.\times \frac{[|n-\eta|+b(n-\eta)]^{(1-\sigma) / p}}{[|x-\xi|+a(x-\xi)]^{(1+\delta \sigma) / q}} \mathrm{~d} x\right\}^{p} \\
& \leq \int_{-\infty}^{\infty} H(x, n) \frac{[|x-\xi|+a(x-\xi)]^{(1+\delta \sigma)(p-1)}}{[|n-\eta|+b(n-\eta)]^{1-\sigma}} f^{p}(x) \mathrm{d} x \\
& \times\left\{\int_{-\infty}^{\infty} H(x, n) \frac{[|n-\eta|+b(n-\eta)]^{(1-\sigma)(q-1)}}{[|x-\xi|+a(x-\xi)]^{1+\delta \sigma}} \mathrm{d} x\right\}^{p-1} \\
&= \frac{\omega^{p-1}(\sigma, n)}{[|n-\eta|+b(n-\eta)]^{p \sigma-1}} \\
& \times \int_{-\infty}^{\infty} H(x, n) \frac{[|x-\xi|+a(x-\xi)]^{(1+\delta \sigma)(p-1)}}{[|n-\eta|+b(n-\eta)]^{1-\sigma}} f^{p}(x) \mathrm{d} x .
\end{aligned}
$$

Then by (8) and the Lebesgue term by term integration theorem (cf. [18]), in view of (7), we derive that

$$
\begin{aligned}
J_{1} & \leq k_{a}^{\frac{1}{q}}(\sigma)\left\{\sum_{|n|=1}^{\infty} \int_{-\infty}^{\infty} g(x, n) \frac{[|x-\xi|+a(x-\xi)]^{(1+\delta \sigma)(p-1)}}{[|n-\eta|+b(n-\eta)]^{1-\sigma}} f^{p}(x) \mathrm{d} x\right\}^{\frac{1}{p}} \\
& =k_{a}^{\frac{1}{q}}(\sigma)\left\{\int_{-\infty}^{\infty} \sum_{|n|=1}^{\infty} g(x, n) \frac{[|x-\xi|+a(x-\xi)]^{(1+\delta \sigma)(p-1)}}{[|n-\eta|+b(n-\eta)]^{1-\sigma}} f^{p}(x) \mathrm{d} x\right\}^{\frac{1}{p}} \\
& =k_{a}^{\frac{1}{q}}(\sigma)\left\{\int_{-\infty}^{\infty} \varpi(\sigma, x)[|x-\xi|+a(x-\xi)]^{p(1+\delta \sigma)-1} f^{p}(x) \mathrm{d} x\right\}^{\frac{1}{p}} .
\end{aligned}
$$

Hence, by (9), we deduce (17).

By Hölder's inequality (cf. [17]), we have

$$
\begin{aligned}
I= & \sum_{|n|=1}^{\infty}\left\{[|n-\eta|+b(n-\eta)]^{\frac{-1}{p}+\sigma} \int_{-\infty}^{\infty} H(x, n) f(x) \mathrm{d} x\right\} \\
& \times\left\{[|n-\eta|+b(n-\eta)]^{\frac{1}{p}-\sigma} b_{n}\right\} \\
\leq & J_{1}\left[\sum_{|n|=1}^{\infty}[|n-\eta|+b(n-\eta)]^{q(1-\sigma)-1} b_{n}^{q}\right]^{\frac{1}{q}} .
\end{aligned}
$$

Then by (17), we get (16). On the other hand, assuming that (16) is valid, we set 


$$
b_{n}:=[|n-\eta|+b(n-\eta)]^{p \sigma-1}\left[\int_{-\infty}^{\infty} H(x, n) f(x) \mathrm{d} x\right]^{p-1}(|n| \in \mathbf{N}) .
$$

Then we obtain that

$$
J_{1}=\left[\sum_{|n|=1}^{\infty}[|n-\eta|+b(n-\eta)]^{q(1-\sigma)-1} b_{n}^{q}\right]^{\frac{1}{p}} .
$$

In view of (22), it follows that $J_{1}<\infty$. If $J_{1}=0$, then (17) is trivially valid; if $J_{1}>0$, then by (16), we have

$$
\begin{aligned}
\sum_{|n|=1}^{\infty}[ & |n-\eta|+b(n-\eta)]^{q(1-\sigma)-1} b_{n}^{q} \\
= & J_{1}^{p}=I<K_{a, b}(\sigma)\left\{\int_{-\infty}^{\infty}[|x-\xi|+a(x-\xi)]^{p(1+\delta \sigma)-1} f^{p}(x) \mathrm{d} x\right\}^{\frac{1}{p}} \\
& \times\left\{\sum_{|n|=1}^{\infty}[|n-\eta|+b(n-\eta)]^{q(1-\sigma)-1} b_{n}^{q}\right\}^{\frac{1}{q}}, \\
& \left\{\sum_{|n|=1}^{\infty}[|n-\eta|+b(n-\eta)]^{q(1-\sigma)-1} b_{n}^{q}\right\}^{\frac{1}{p}} \\
= & J_{1}<K_{a, b}(\sigma)\left\{\int_{-\infty}^{\infty}[|x-\xi|+a(x-\xi)]^{p(1+\delta \sigma)-1} f^{p}(x) \mathrm{d} x\right\}^{\frac{1}{p}},
\end{aligned}
$$

namely (17) holds, which is equivalent to (16).

Similarly to as we obtained (22), by Hölder's inequality, we have 


$$
\begin{aligned}
& {\left[\sum_{|n|=1}^{\infty} H(x, n) b_{n}\right]^{q} } \\
&=\left\{\sum_{|n|=1}^{\infty} H(x, n) \frac{[|x-\xi|+a(x-\xi)]^{(1+\delta \sigma) / q}}{[|n-\eta|+b(n-\eta)]^{(1-\sigma) / p}}\right. \\
&\left.\quad \times \frac{[|n-\eta|+b(n-\eta)]^{(1-\sigma) / p}}{[|x-\xi|+a(x-\xi)]^{(1+\delta \sigma) / q}} b_{n}\right\}^{q} \\
& \leq\left\{\sum_{|n|=1}^{\infty} H(x, n) \frac{[|x-\xi|+a(x-\xi)]^{(1+\delta \sigma)(p-1)}}{[|n-\eta|+b(n-\eta)]^{1-\sigma}}\right\}^{q-1} \\
& \quad \times \sum_{|n|=1}^{\infty} H(x, n) \frac{[|n-\eta|+b(n-\eta)]^{(1-\sigma)(q-1)}}{[|x-\xi|+a(x-\xi)]^{1+\delta \sigma}} b_{n}^{q} \\
&=\frac{[|x-\xi|+a(x-\xi)]^{-q \delta \sigma-1}}{[\varpi(\sigma, x))^{q-1}} \\
& \times \sum_{|n|=1}^{\infty} H(x, n) \frac{[|n-\eta|+b(n-\eta)]^{(1-\sigma)(q-1)}}{[|x-\xi|+a(x-\xi)]^{1+\delta \sigma}} b_{n}^{q} .
\end{aligned}
$$

By (9) and the Lebesgue term by term theorem, we have

$$
\begin{aligned}
J_{2} & <k_{a}^{\frac{1}{p}}(\sigma)\left\{\int_{-\infty}^{\infty} \sum_{|n|=1}^{\infty} H(x, n) \frac{[|n-\eta|+b(n-\eta)]^{(1-\sigma)(q-1)}}{[|x-\xi|+a(x-\xi)]^{1+\delta \sigma}} b_{n}^{q} \mathrm{~d} x\right\}^{\frac{1}{q}} \\
& =k_{a}^{\frac{1}{p}}(\sigma)\left\{\sum_{|n|=1}^{\infty} \omega(\sigma, n)[|n-\eta|+b(n-\eta)]^{q(1-\sigma)-1} b_{n}^{q}\right\}^{\frac{1}{q}} .
\end{aligned}
$$

Hence, by (8), we deduce (18).

We have proved that (16) is satisfied. Setting

$$
f(x):=[|x-\xi|+a(x-\xi)]^{-q \delta \sigma-1}\left[\sum_{|n|=1}^{\infty} H(x, n) b_{n}\right]^{q-1}(x \in \mathbf{R} \backslash\{\xi\}),
$$

it then follows that

$$
J_{2}=\left\{\int_{-\infty}^{\infty}[|x-\xi|+a(x-\xi)]^{p(1+\delta \sigma)-1} f^{p}(x) \mathrm{d} x\right\}^{\frac{1}{q}},
$$

and in view of (24), we obtain that $J_{2}<\infty$. If $J_{2}=0$, then ( 18) is trivially valid; if $J_{2}>0$, then by (16) we have 


$$
\begin{aligned}
& \int_{-\infty}^{\infty}[|x-\xi|+a(x-\xi)]^{p(1+\delta \sigma)-1} f^{p}(x) \mathrm{d} x \\
& =J_{2}^{q}=I<K_{a, b}(\sigma)\left\{\int_{-\infty}^{\infty}[|x-\xi|+a(x-\xi)]^{p(1+\delta \sigma)-1} f^{p}(x) \mathrm{d} x\right\}^{\frac{1}{p}} \\
& \quad \times\left\{\sum_{|n|=1}^{\infty}[|n-\eta|+b(n-\eta)]^{q(1-\sigma)-1} b_{n}^{q}\right\}^{\frac{1}{q}}, \\
& \left\{\int_{-\infty}^{\infty}[|x-\xi|+a(x-\xi)]^{p(1+\delta \sigma)-1} f^{p}(x) \mathrm{d} x\right\}^{\frac{1}{q}} \\
& =J_{2}<K_{a, b}(\sigma)\left\{\sum_{|n|=1}^{\infty}[|n-\eta|+b(n-\eta)]^{q(1-\sigma)-1} b_{n}^{q}\right\}^{\frac{1}{q}}
\end{aligned}
$$

namely, (18) follows.

On the other hand, assuming that (18) is valid, by Hölder's inequality (cf. [17]) and similarly to as we obtained (23), we have

$$
I \leq\left\{\int_{-\infty}^{\infty}[|x-\xi|+a(x-\xi)]^{p(1+\delta \sigma)-1} f^{p}(x) \mathrm{d} x\right\}^{\frac{1}{p}} J_{2} .
$$

Then by (18) we derive (16), which is equivalent to (18).

Therefore, inequalities (16), (17) and (18) are equivalent.

This completes the proof of the theorem.

Theorem 3.2 With regards to the assumptions of Theorem 1, the constant factor $K_{a, b}(\sigma)$ in (16), (17) and (18) is the best possible.

Proof For $0<\varepsilon<q \sigma$, we set $\tilde{\sigma}=\sigma-\frac{\varepsilon}{q}(\in(0, \lambda))$,

$$
\widetilde{f}(x):=\left\{\begin{array}{c}
\frac{1}{[|x-\xi|+a(x-\xi)]^{\delta\left(\sigma+\frac{\varepsilon}{p}\right)+1}}, x \in E_{\delta}, \\
0, x \in \mathbf{R} \backslash E_{\delta},
\end{array}\right.
$$

and

$$
\widetilde{b}_{n}:=[|n-\eta|+b(n-\eta)]^{\left(\sigma-\frac{\varepsilon}{q}\right)-1},|n| \in \mathbf{N} .
$$

Then by (12) and (14), we obtain that 


$$
\begin{aligned}
\widetilde{I}_{1}:= & \left\{\int_{-\infty}^{\infty}[|x-\xi|+a(x-\xi)]^{p(1+\delta \sigma)-1} \widetilde{f}^{p}(x) \mathrm{d} x\right\}^{\frac{1}{p}} \\
& \times\left\{\sum_{|n|=1}^{\infty}[|n-\eta|+b(n-\eta)]^{q(1-\sigma)-1} \widetilde{b}_{n}^{q}\right\}^{\frac{1}{q}} \\
= & \left\{\int_{-\infty}^{\infty} \frac{\mathrm{d} x}{[|x-\xi|+a(x-\xi)]^{\delta \varepsilon+1}}\right\}^{\frac{1}{p}} \\
& \times\left\{\sum_{|n|=1}^{\infty} \frac{1}{[|n-\eta|+b(n-\eta)]^{\varepsilon+1}}\right\}^{\frac{1}{q}} \\
\leq & \frac{1}{\varepsilon}\left(\frac{2}{1-a^{2}}\right)^{\frac{1}{p}}\left[\left(\frac{2}{1-b^{2}}+o_{1}(1)\right)\left(1+o_{2}(1)\right)\right]^{\frac{1}{q}} .
\end{aligned}
$$

By (9), we also have that

$$
\begin{aligned}
\widetilde{I}: & =\sum_{|n|=1}^{\infty} \int_{-\infty}^{\infty} H(x, n) \tilde{f}(x) \tilde{b}_{n} \mathrm{~d} x \\
& =\int_{E_{\delta}} \sum_{|n|=1}^{\infty} H(x, n) \frac{[|x-\xi|+a(x-\xi)]^{-\delta(\widetilde{\sigma}+\varepsilon)-1}}{[|n-\eta|+b(n-\eta)]^{1-\tilde{\sigma}}} \mathrm{d} x \\
& =\int_{E_{\delta}} \frac{\varpi(\tilde{\sigma}, x)}{[|x-\xi|+a(x-\xi)]^{\delta \varepsilon+1}} \mathrm{~d} x \\
\geq & k_{b}(\tilde{\sigma}) \int_{E_{\delta}} \frac{1-\theta(\tilde{\sigma}, x)}{[|x-\xi|+a(x-\xi)]^{\delta \varepsilon+1}} \mathrm{~d} x \\
= & k_{b}(\widetilde{\sigma})\left\{\int_{E_{\delta}} \frac{1}{[|x-\xi|+a(x-\xi)]^{\delta \varepsilon+1}} \mathrm{~d} x\right. \\
& \left.-\int_{E_{\delta}} \frac{1}{O\left([|x-\xi|+a(x-\xi)]^{\delta\left(\sigma+\frac{\varepsilon}{p}-\lambda \kappa\right)+1}\right)} \mathrm{d} x\right\} \\
= & \frac{1}{\varepsilon} k_{b}\left(\sigma-\frac{\varepsilon}{q}\right)\left(\frac{2}{1-a^{2}}-\varepsilon O(1)\right)\left(0<\kappa<\frac{\sigma-\varepsilon / q}{\lambda}\right) .
\end{aligned}
$$

If the constant factor $K_{a, b}(\sigma)$ in (16) is not the best possible, then there exists a positive number $k$, with $K_{a, b}(\sigma)>k$, such that (16) is valid when we replace $K_{a, b}(\sigma)$ by $k$. Then in particular, we have $\varepsilon \tilde{I}<\varepsilon \widetilde{I}_{1}$, namely,

$$
\begin{aligned}
& k_{b}\left(\sigma-\frac{\varepsilon}{q}\right)\left(\frac{2}{1-a^{2}}-\varepsilon O(1)\right) \\
& \quad<k \cdot\left(\frac{2}{1-a^{2}}\right)^{\frac{1}{p}}\left[\left(\frac{2}{1-b^{2}}+o_{1}(1)\right)\left(1+o_{2}(1)\right)\right]^{\frac{1}{q}} .
\end{aligned}
$$

It follows that 


$$
k_{b}(\sigma) \frac{2}{1-a^{2}} \leq k\left(\frac{2}{1-a^{2}}\right)^{\frac{1}{p}}\left(\frac{2}{1-b^{2}}\right)^{\frac{1}{q}}\left(\varepsilon \rightarrow 0^{+}\right),
$$

that is,

$$
K_{a, b}(\sigma)=\frac{2\left[\frac{\pi}{\lambda \sin \left(\frac{\pi \sigma}{\lambda}\right)}\right]^{2}}{\left(1-a^{2}\right)^{1 / q}\left(1-b^{2}\right)^{1 / p}} \leq k .
$$

This is a contradiction. Hence, the constant factor $K_{a, b}(\sigma)$ in (16) is the best possible.

The constant factor $K_{a, b}(\sigma)$ in (17) (resp. (18)) is still the best possible. Otherwise, we would reach a contradiction by (23) (resp. (25)) that the constant factor $K_{a, b}(\sigma)$ in (16) is not the best possible.

This completes the proof of the theorem.

\section{Operator expressions}

Suppose that $p>1, \frac{1}{p}+\frac{1}{q}=1$. We set the following functions:

$$
\begin{aligned}
& \Phi(x):=[|x-\xi|+a(x-\xi)]^{p(1+\delta \sigma)-1}, \\
& \Psi(n):=[|n-\eta|+b(n-\eta)]^{q(1-\sigma)-1},
\end{aligned}
$$

wherefrom,

$$
\begin{aligned}
& \Phi^{1-q}(x)=[|x-\xi|+a(x-\xi)]^{-q \delta \sigma-1}, \\
& \Psi^{1-p}(n)=[|n-\eta|+b(n-\eta)]^{p \sigma-1}(x \in \mathbf{R} \backslash\{\xi\},|n| \in \mathbf{N}) .
\end{aligned}
$$

Define the following real weight normed linear spaces:

$$
\begin{aligned}
L_{p, \Phi}(\mathbf{R}) & :=\left\{f ;\left.|| f\right|_{p, \Phi}:=\left(\int_{-\infty}^{\infty} \Phi(x)|f(x)|^{p} \mathrm{~d} x\right)^{\frac{1}{p}}<\infty\right\}, \\
L_{q, \Phi^{1-q}}(\mathbf{R}) & :=\left\{h ;\left.|| h\right|_{q, \Phi^{1-q}}:=\left(\int_{-\infty}^{\infty} \Phi^{1-q}(x)|h(x)|^{q} \mathrm{~d} x\right)^{\frac{1}{q}}<\infty\right\}, \\
l_{q, \Psi} & :=\left\{b=\left\{b_{n}\right\}_{|n|=1}^{\infty} ;\left.|| b\right|_{q, \Psi}:=\left(\sum_{|n|=1}^{\infty} \Psi(n)\left|b_{n}\right|^{q}\right)^{\frac{1}{q}}<\infty\right\} \\
l_{p, \Psi 1-p} & :=\left\{\begin{array}{l}
\left.c=\left\{c_{n}\right\}_{|n|=1}^{\infty} ;|| c||_{p, \Psi^{1-p}}:=\left(\sum_{|n|=1}^{\infty} \Psi^{1-p}(n)\left|c_{n}\right|^{p}\right)^{\frac{1}{p}}<\infty\right\} .
\end{array}\right.
\end{aligned}
$$


(a) In view of Theorem 1 , for $f \in L_{p, \Phi}(\mathbf{R})$, setting

$$
H^{(1)}(n):=\int_{-\infty}^{\infty} H(x, n)|f(x)| \mathrm{d} x(|n| \in \mathbf{N}),
$$

by (17), we have

$$
\left\|H^{(1)}\right\|_{p, \Psi 1-p}=\left[\sum_{|n|=1}^{\infty} \Psi^{1-p}(n)\left(H^{(1)}(n)\right)^{p}\right]^{\frac{1}{p}}<K_{a, b}(\sigma)\|f\|_{p, \Phi}<\infty
$$

namely, $H^{(1)} \in l_{p, \Psi^{1-p}}$.

Definition 4.1 Define a Hilbert-type operator in the whole plane

$$
T^{(1)}: L_{p, \Phi}(\mathbf{R}) \rightarrow l_{p, \Psi^{1-p}}
$$

as follows:

For any $f \in L_{p, \Phi}(\mathbf{R})$, there exists a unique representation

$$
T^{(1)} f=H^{(1)} \in l_{p, \Psi^{1-p}},
$$

satisfying

$$
\left(T^{(1)} f\right)(n)=H^{(1)}(n),
$$

for any $|n| \in \mathbf{N}$.

In view of (26), it follows that

$$
\left\|T^{(1)} f\right\|_{p, \Psi^{1-p}}=\left\|H^{(1)}\right\|_{p, \Psi^{1-p}} \leq K_{a, b}\|f\|_{p, \Phi},
$$

and then the operator $T^{(1)}$ is bounded satisfying

$$
\left\|T^{(1)}\right\|=\sup _{f(\neq \theta) \in L_{p, \Phi}(\mathbf{R})} \frac{\left\|T^{(1)} f\right\|_{p, \Psi^{1-p}}}{\|f\|_{p, \Phi}} \leq K_{a, b}(\sigma) .
$$

Since the constant factor $K_{a, b}(\sigma)$ in (26) is the best possible, we have

$$
\left\|T^{(1)}\right\|=K_{a, b}(\sigma)=\frac{2\left[\frac{\pi}{\lambda \sin \left(\frac{\pi \sigma}{\lambda}\right)}\right]^{2}}{\left(1-a^{2}\right)^{1 / q}\left(1-b^{2}\right)^{1 / p}} .
$$

If we define the formal inner product of $T^{(1)} f$ and $b\left(\in l_{q, \Psi}\right)$ as follows:

$$
\left(T^{(1)} f, b\right):=\sum_{|n|=1}^{\infty}\left(\int_{-\infty}^{\infty} H(x, n) f(x) \mathrm{d} x\right) b_{n}
$$

we can then rewrite (16) and (17) as follows: 


$$
\left(T^{(1)} f, b\right)<\left\|T^{(1)}\right\| \cdot\|f\|_{p, \Psi}\|b\|_{q, \Phi},\left\|T^{(1)} f\right\|_{p, \Psi 1-p}<\left\|T^{(1)}\right\| \cdot\|f\|_{p, \Phi} .
$$

(b) In view of Theorem 1 , for $b \in l_{q, \Psi}$, setting

$$
H^{(2)}(x):=\sum_{|n|=1}^{\infty} H(x, n) b_{n}(x \in \mathbf{R}),
$$

then by (18) we have

$$
\left\|H^{(2)}\right\|_{q, \Phi^{1-q}}=\left[\int_{-\infty}^{\infty} \Phi^{1-q}(x)\left(H^{(2)}(x)\right)^{q} \mathrm{~d} x\right]^{\frac{1}{q}}<K_{a, b}(\sigma)\|b\|_{q, \Psi}<\infty,
$$

namely $H^{(2)} \in L_{q, \Psi 1-q}(\mathbf{R})$.

Definition 4.2 Define a Hilbert-type operator in the whole plane

$$
T^{(2)}: l_{q, \Psi} \rightarrow L_{q, \Psi^{1-q}}(\mathbf{R})
$$

as follows:

For any $b \in l_{q, \Psi}$, there exists a unique representation

$$
T^{(2)} b=H^{(2)} \in L_{q, \Psi 1-q}(\mathbf{R}),
$$

satisfying

$$
\left(T^{(2)} b\right)(x)=H^{(2)}(x)
$$

for any $x \in \mathbf{R}$.

In view of (29), we have

$$
\left\|T^{(2)} b\right\|_{q, \Phi^{1-q}}=\left\|H^{(2)}\right\|\left\|_{q, \Phi^{1-q}} \leq K_{a, b}(\sigma)\right\| b \|_{q, \Psi},
$$

and then the operator $T^{(2)}$ is bounded satisfying

$$
\left\|T^{(2)}\right\|=\sup _{b(\neq \theta) \in l_{q, \Psi}} \frac{\left\|T^{(2)} b\right\|_{q, \Phi^{1-q}}}{\|b\|_{q, \Psi}} \leq K_{a, b}(\sigma) .
$$

Since the constant factor $K_{a, b}(\sigma)$ in (29) is the best possible, we have

$$
\left\|T^{(2)}\right\|=K_{a, b}(\sigma)=\left\|T^{(1)}\right\| .
$$

If we define the formal inner product of $T^{(2)} b$ and $f\left(\in L_{p, \Phi}(\mathbf{R})\right)$ as follows:

$$
\left(T^{(2)} b, f\right):=\int_{-\infty}^{\infty} \sum_{|n|=1}^{\infty} H(x, n) b_{n} f(x) \mathrm{d} x,
$$

then we can rewrite (16) and (18) in the following manner: 


$$
\left(T^{(2)} b, f\right)<\left\|T^{(2)}\right\| \cdot|| f\left\|_{p, \Psi}\right\| b\left\|_{q, \Phi},\right\| T^{(2)} b\left\|_{q, \Phi^{1-q}}<\right\| T^{(2)}\|\cdot\| b \|_{q, \Psi}
$$

Remark 4.3 (i) For $\xi=\eta=0, \delta=1$, (19) reduces to (5). If $f(-x)=f(x)(x>0)$, $b_{-n}=b_{n}(n \in \mathbf{N})$, then (5) reduces to the following half-discrete Hilbert-type inequality (cf. [40]):

$$
\begin{aligned}
& \sum_{n=1}^{\infty} \int_{0}^{\infty} \frac{\ln \left(\frac{n}{x}\right)}{\left(\frac{n}{x}\right)^{\lambda}-1} f(x) b_{n} \mathrm{~d} x \\
& \quad<\left[\frac{\pi}{\lambda \sin \left(\frac{\pi \sigma}{\lambda}\right)}\right]^{2}\left[\int_{0}^{\infty} x^{p(1+\sigma)-1} f^{p}(x) \mathrm{d} x\right]^{\frac{1}{p}}\left[\sum_{n=1}^{\infty} n^{q(1-\sigma)-1} b_{n}^{q}\right]^{\frac{1}{q}} .
\end{aligned}
$$

(ii) For $\delta=1$, replacing $[|x-\xi|+a(x-\xi)]^{\lambda} f(x)$ to $f(x)$, (16) reduces to the following particular inequality with homogeneous kernel of degree $-\lambda$ :

$$
\begin{aligned}
& \sum_{|n|=1}^{\infty} \int_{-\infty}^{\infty} \frac{\ln \left(\frac{|n-\eta|+b(n-\eta)}{|x-\xi|+a(x-\xi)}\right) f(x) b_{n}}{[|n-\eta|+b(n-\eta)]^{\lambda}-[|x-\xi|+a(x-\xi)]^{\lambda}} \mathrm{d} x \\
& <K_{a, b}(\sigma)\left[\int_{-\infty}^{\infty}[|x-\xi|+a(x-\xi)]^{p(1-\mu)-1} f^{p}(x) \mathrm{d} x\right]^{\frac{1}{p}} \\
& \quad \times\left[\sum_{|n|=1}^{\infty}[|n-\eta|++b(n-\eta)]^{q(1-\sigma)-1} b_{n}^{q}\right]^{\frac{1}{q}},
\end{aligned}
$$

(iii) For $\delta=-1,(16)$ reduces to the following particular inequality with nonhomogeneous kernel:

$$
\begin{aligned}
& \sum_{|n|=1}^{\infty} \int_{-\infty}^{\infty} \frac{\ln \{[x-\xi \mid+a(x-\xi)][|n-\eta|+b(n-\eta)]\} f(x)}{\{[|n-\eta|+b(n-\eta)][x-\xi \mid+a(x-\xi)]\}^{\lambda}-1} b_{n} \mathrm{~d} x \\
& <K_{a, b}(\sigma)\left\{\int_{-\infty}^{\infty}[|x-\xi|+a(x-\xi)]^{p(1-\sigma)-1} f^{p}(x) \mathrm{d} x\right\}^{\frac{1}{p}} \\
& \quad \times\left\{\sum_{|n|=1}^{\infty}[|n-\eta|+b(n-\eta)]^{q(1-\sigma)-1} b_{n}^{q}\right\}^{\frac{1}{q}} .
\end{aligned}
$$

The constant factors in the above inequalities are the best possible. 


\section{Some equivalent reverses}

In the sequel, for the cases when $0<p<1$ and $p<0$, we still use $\|b\|_{q, \Phi}$ and $\|f\|_{p, \Psi}$ as the formal symbols.

Theorem 5.1 Suppose that $0<p<1, \frac{1}{p}+\frac{1}{q}=1$. If $f(x), b_{n} \geq 0$, satisfying $0<\|f\|_{p, \Psi},\|b\|_{q, \Phi}<\infty$, then we have the following equivalent inequalities:

$$
\begin{aligned}
I & =\sum_{|n|=1}^{\infty} \int_{-\infty}^{\infty} H(x, n) f(x) b_{n} \mathrm{~d} x>K_{a, b}(\sigma) \\
& \times\left\{\int_{-\infty}^{\infty}(1-\theta(\sigma, x))[|x-\xi|+a(x-\xi)]^{p(1+\delta \sigma)-1} f^{p}(x) \mathrm{d} x\right\}^{\frac{1}{p}}|| b||_{q, \Phi}, \\
J_{1} & =\left\{\sum_{|n|=1}^{\infty}[|n-\eta|+b(n-\eta)]^{p \sigma-1}\left(\int_{-\infty}^{\infty} H(x, n) f(x) \mathrm{d} x\right)^{p}\right\}^{\frac{1}{p}} \\
& >K_{a, b}(\sigma)\left\{\int_{-\infty}^{\infty}(1-\theta(\sigma, x))[|x-\xi|+a(x-\xi)]^{p(1+\delta \sigma)-1} f^{p}(x) \mathrm{d} x\right\}^{\frac{1}{p}}, \\
& \widetilde{J}_{2}:=\left\{\int_{-\infty}^{\infty} \frac{(1-\theta(\sigma, x))^{1-q}}{[|x-\xi|+a(x-\xi)]^{q \delta \sigma+1}}\left(\sum_{|n|=1}^{\infty} H(x, n) b_{n}\right)^{q} \mathrm{~d} x\right\}^{\frac{1}{q}} \\
& >K_{a, b}(\sigma)|| b||_{q, \Phi},
\end{aligned}
$$

where the constant factor $K_{a, b}(\sigma)$ is the best possible.

Proof By the reverse Hölder inequality (cf. [17]) and (6), we obtain that

$$
\begin{aligned}
{\left[\int_{-\infty}^{\infty} H(x, n) f(x) \mathrm{d} x\right]^{p} \geq } & \frac{\omega^{p-1}(\sigma, n)}{[|n-\eta|+b(n-\eta)]^{p \sigma-1}} \\
& \times \int_{-\infty}^{\infty} H(x, n) \frac{[|x-\xi|+a(x-\xi)]^{(1+\delta \sigma)(p-1)}}{[|n-\eta|+b(n-\eta)]^{1-\sigma}} f^{p}(x) \mathrm{d} x .
\end{aligned}
$$

Then by (8) and the Lebesgue term by term integration theorem (cf. [18]), in view of (7), we derive that

$$
J_{1} \geq k_{a}^{\frac{1}{q}}(\sigma)\left\{\int_{-\infty}^{\infty} \varpi(\sigma, x)[|x-\xi|+a(x-\xi)]^{p(1+\delta \sigma)-1} f^{p}(x) \mathrm{d} x\right\}^{\frac{1}{p}} .
$$

Hence, by (9), we deduce (36).

By the reverse Hölder inequality (cf. [17]), we have 


$$
I \geq J_{1}\left\{\sum_{|n|=1}^{\infty}[|n-\eta|+b(n-\eta)]^{q(1-\sigma)-1} b_{n}^{q}\right\}^{\frac{1}{q}} .
$$

Then by (36), we deduce (35).

On the other hand, assuming that (35) is satisfied, we set

$$
b_{n}:=[|n-\eta|+b(n-\eta)]^{p \sigma-1}\left[\int_{-\infty}^{\infty} H(x, n) f(x) \mathrm{d} x\right]^{p-1}(|n| \in \mathbf{N}) .
$$

Then we obtain that

$$
J_{1}=\left[\sum_{|n|=1}^{\infty}[|n-\eta|+b(n-\eta)]^{q(1-\sigma)-1} b_{n}^{q}\right]^{\frac{1}{p}} .
$$

In view of (38), it follows that $J_{1}>0$. If $J_{1}=\infty$, then (36) is trivially valid; if $J_{1}<\infty$, then by (35), we have

$$
\begin{aligned}
& \|b\|_{q, \Phi}^{q}=J_{1}^{p}=I \\
& \quad>K_{a, b}(\sigma)\left\{\int_{-\infty}^{\infty}(1-\theta(\sigma, x))[|x-\xi|+a(x-\xi)]^{p(1+\delta \sigma)-1} f^{p}(x) \mathrm{d} x\right\}^{\frac{1}{p}}\|b\|_{q, \Phi}, \\
& \|b\|_{q, \Phi}^{q-1}=J_{1} \\
& \quad>K_{a, b}(\sigma)\left\{\int_{-\infty}^{\infty}(1-\theta(\sigma, x))[|x-\xi|+a(x-\xi)]^{p(1+\delta \sigma)-1} f^{p}(x) \mathrm{d} x\right\}^{\frac{1}{p}},
\end{aligned}
$$

namely (36) holds true, which is equivalent to (35).

Similarly to as we obtained (38), we derive that

$$
\widetilde{J}_{2}>k_{a}^{\frac{1}{p}}(\sigma)\left\{\sum_{|n|=1}^{\infty} \omega(\sigma, n)[|n-\eta|+b(n-\eta)]^{q(1-\sigma)-1} b_{n}^{q}\right\}^{\frac{1}{q}} .
$$

Hence, by (8), we deduce (37). We have proved that (35) is valid. Setting

$$
f(x):=\frac{(1-\theta(\sigma, x))^{1-q}}{[|x-\xi|+a(x-\xi)]^{q \delta \sigma+1}}\left[\sum_{|n|=1}^{\infty} H(x, n) b_{n}\right]^{q-1}(x \in \mathbf{R} \backslash\{\xi\}),
$$

then it follows that

$$
\widetilde{J}_{2}=\left\{\int_{-\infty}^{\infty}(1-\theta(\sigma, x))[|x-\xi|+a(x-\xi)]^{p(1+\delta \sigma)-1} f^{p}(x) \mathrm{d} x\right\}^{\frac{1}{q}},
$$

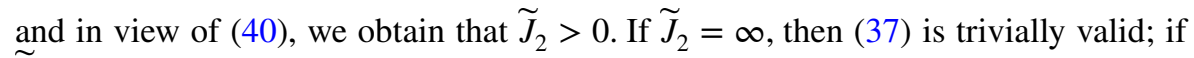
$\widetilde{J}_{2}<\infty$, then by (35), we have 


$$
\begin{aligned}
\int_{-\infty}^{\infty} & (1-\theta(\sigma, x))[|x-\xi|+a(x-\xi)]^{p(1+\delta \sigma)-1} f^{p}(x) \mathrm{d} x=\widetilde{J}_{2}^{q}=I \\
> & K_{a, b}(\sigma)\left\{\int_{-\infty}^{\infty}(1-\theta(\sigma, x))[|x-\xi|+a(x-\xi)]^{p(1+\delta \sigma)-1} f^{p}(x) \mathrm{d} x\right\}^{\frac{1}{p}}\|b\|_{q, \Phi}, \\
& \left\{\int_{-\infty}^{\infty}(1-\theta(\sigma, x))[|x-\xi|+a(x-\xi)]^{p(1+\delta \sigma)-1} f^{p}(x) \mathrm{d} x\right\}^{\frac{1}{q}} \\
= & \widetilde{J}_{2}>K_{a, b}(\sigma)\|b\|_{q, \Phi},
\end{aligned}
$$

namely, (37) follows.

On the other hand, assuming that (37) is satisfied, by the reverse Hölder inequality (cf. [17]), we obtain

$$
I \geq\left\{\int_{-\infty}^{\infty}(1-\theta(\sigma, x))[|x-\xi|+a(x-\xi)]^{p(1+\delta \sigma)-1} f^{p}(x) \mathrm{d} x\right\}^{\frac{1}{p}} \widetilde{J}_{2} .
$$

Then by (37), we derive (15), which is equivalent to (37).

Therefore, inequalities (35), (36) and (37) are equivalent.

For $0<\varepsilon<p(\lambda-\sigma)$, we set $\widetilde{\sigma}=\sigma+\frac{\varepsilon}{p}(<\lambda)$,

$$
\tilde{f}(x):=\left\{\begin{array}{c}
\frac{1}{[|x-\xi|+a(x-\xi)]^{\delta\left(\sigma+\frac{\varepsilon}{p}\right)+1}}, \quad x \in E_{\delta}, \\
0, \quad x \in \mathbf{R} \backslash E_{\delta},
\end{array}\right.
$$

and

$$
\widetilde{b}_{n}:=[|n-\eta|+b(n-\eta)]^{\left(\sigma-\frac{\varepsilon}{q}\right)-1}, \quad|n| \in \mathbf{N} .
$$

Then by (12) and (14), we find 


$$
\begin{aligned}
\widetilde{I}_{1}:= & \left\{\int_{-\infty}^{\infty}(1-\theta(\sigma, x))[|x-\xi|+a(x-\xi)]^{p(1+\delta \sigma)-1} \widetilde{f}^{p}(x) \mathrm{d} x\right\}^{\frac{1}{p}} \\
& \times\left\{\sum_{|n|=1}^{\infty}[|n-\eta|+b(n-\eta)]^{q(1-\sigma)-1} \widetilde{b}_{n}^{q}\right\}^{\frac{1}{q}} \\
= & \left\{\int_{-\infty}^{\infty} \frac{(1-\theta(\sigma, x)) \mathrm{d} x}{[|x-\xi|+a(x-\xi)]^{\delta \varepsilon+1}}\right\}^{\frac{1}{p}}\left\{\sum_{|n|=1}^{\infty} \frac{1}{[|n-\eta|+b(n-\eta)]^{\varepsilon+1}}\right\}^{\frac{1}{q}} \\
= & \left.\left\{\frac{1}{\varepsilon} \frac{2}{1-a^{2}}-\int_{-\infty}^{\infty} \frac{1}{[|x-\xi|+a(x-\xi)]^{\delta[\sigma-\lambda \kappa+\varepsilon]+1}}\right\}^{\frac{1}{p}}\right\}^{\frac{1}{q}} \\
& \times\left\{\sum_{|n|=1}^{\infty} \frac{1}{[|n-\eta|+b(n-\eta)]^{\varepsilon+1}}\right\}^{\frac{1}{p}}\left[\left(\frac{2}{1-b^{2}}+o_{1}(1)\right)\left(1+o_{2}(1)\right)\right]^{\frac{1}{q}}\left(0<\kappa<\frac{\sigma}{\lambda}\right) . \\
= & \frac{1}{\varepsilon}\left(\frac{2}{1-a^{2}}-\varepsilon O(1)\right)^{\frac{2}{p}}
\end{aligned}
$$

By (9), we also have

$$
\begin{aligned}
\widetilde{I} & :=\sum_{|n|=1}^{\infty} \int_{-\infty}^{\infty} H(x, n) \tilde{f}(x) \tilde{b}_{n} \mathrm{~d} x \\
& =\sum_{|n|=1}^{\infty} \int_{E_{\delta}} H(x, n) \frac{[|n-\eta|+b(n-\eta)]^{\left(\sigma-\frac{\varepsilon}{q}\right)-1}}{[|x-\xi|+a(x-\xi)]^{\delta\left(\sigma+\frac{\varepsilon}{p}\right)+1}} \mathrm{~d} x \\
& \leq \sum_{|n|=1}^{\infty} \int_{-\infty}^{\infty} H(x, n) \frac{[|n-\eta|+b(n-\eta)]^{(\widetilde{\sigma}-\varepsilon)-1}}{[|x-\xi|+a(x-\xi)]^{\delta \widetilde{\sigma}+1}} \mathrm{~d} x \\
& =\sum_{|n|=1}^{\infty} \frac{\omega(\widetilde{\sigma}, n)}{[|n-\eta|+b(n-\eta)]^{\varepsilon+1}} \\
& =k_{a}(\tilde{\sigma}) \sum_{|n|=1}^{\infty} \frac{1}{[|n-\eta|+b(n-\eta)]^{\varepsilon+1}} \\
& =\frac{1}{\varepsilon} k_{a}(\tilde{\sigma})\left(\frac{2}{1-b^{2}}+o_{1}(1)\right)\left(1+o_{2}(1)\right)
\end{aligned}
$$

If the constant factor $K_{a, b}(\sigma)$ in (36) is not the best possible, then there exists a positive number $k$, with $K_{a, b}(\sigma)<k$, such that (36) is satisfied when we replace $K_{a, b}(\sigma)$ by $k$. Then in particular, we have $\varepsilon \tilde{I}>\varepsilon k \widetilde{I}_{1}$, namely

$$
\begin{aligned}
& k_{a}\left(\sigma+\frac{\varepsilon}{p}\right)\left(\frac{2}{1-b^{2}}+o_{1}(1)\right)\left(1+o_{2}(1)\right) \\
> & k \cdot\left(\frac{2}{1-a^{2}}-\varepsilon O(1)\right)^{\frac{1}{p}}\left[\left(\frac{2}{1-b^{2}}+o_{1}(1)\right)\left(1+o_{2}(1)\right)\right]^{\frac{1}{q}} .
\end{aligned}
$$


It follows that

$$
k_{a}(\sigma) \frac{2}{1-b^{2}} \geq k\left(\frac{2}{1-a^{2}}\right)^{\frac{1}{p}}\left(\frac{2}{1-b^{2}}\right)^{\frac{1}{q}}\left(\varepsilon \rightarrow 0^{+}\right),
$$

namely

$$
K_{a, b}(\sigma)=\frac{2\left[\frac{\pi}{\lambda \sin \left(\frac{\pi \sigma}{\lambda}\right)}\right]^{2}}{\left(1-a^{2}\right)^{1 / q}\left(1-b^{2}\right)^{1 / p}} \geq k .
$$

This is a contradiction. Hence, the constant factor $K_{a, b}(\sigma)$ in (35) is the best possible.

The constant factor $K_{a, b}(\sigma)$ in (36) ((37)) is still the best possible. Otherwise, we would reach a contradiction by (39) ((41)) that the constant factor $K_{a, b}(\sigma)$ in (35) is not the best possible.

This completes the proof of the theorem.

Theorem 5.2 Suppose that $\quad p<0, \quad \frac{1}{p}+\frac{1}{q}=1 . \quad$ If $f(x), b_{n} \geq 0$, $0<\|f\|_{p, \Psi},\|b\|_{q, \Phi}<\infty$. Then we have the following equivalent inequalities:

$$
\begin{gathered}
I=\sum_{|n|=1}^{\infty} \int_{-\infty}^{\infty} H(x, n) f(x) b_{n} \mathrm{~d} x>K_{a, b}(\sigma)|| f\left|\left\|_{p, \Psi}|| b\right\|_{q, \Phi},\right. \\
J_{1}=\left\{\sum_{|n|=1}^{\infty}[|n-\eta|+b(n-\eta)]^{p \sigma-1}\left(\int_{-\infty}^{\infty} H(x, n) f(x) \mathrm{d} x\right)^{p}\right\}^{\frac{1}{p}} \\
>K_{a, b}(\sigma)|| f \|_{p, \Psi}, \\
J_{2}=\left\{\int_{-\infty}^{\infty} \frac{1}{[|x-\xi|+a(x-\xi)]^{q \delta \sigma+1}}\left(\sum_{|n|=1}^{\infty} H(x, n) b_{n}\right)^{q} \mathrm{~d} x\right\}^{\frac{1}{q}} \\
>K_{a, b}(\sigma)\|\mid b\|_{q, \Phi},
\end{gathered}
$$

where the constant factor $K_{a, b}(\sigma)$ is the best possible.

Proof By the reverse Hölder's inequality (cf. [17]) and (6), we obtain that

$$
\begin{aligned}
{\left[\int_{-\infty}^{\infty} H(x, n) f(x) \mathrm{d} x\right]^{p} \leq } & \frac{\omega^{p-1}(\sigma, n)}{[|n-\eta|+b(n-\eta)]^{p \sigma-1}} \\
& \times \int_{-\infty}^{\infty} H(x, n) \frac{[|x-\xi|+a(x-\xi)]^{(1+\delta \sigma)(p-1)}}{[|n-\eta|+b(n-\eta)]^{1-\sigma}} f^{p}(x) \mathrm{d} x .
\end{aligned}
$$

Then by (8) and the Lebesgue term by term integration theorem (cf. [18]), in view of (7), we deduce that 


$$
J_{1} \geq k_{a}^{\frac{1}{q}}(\sigma)\left\{\int_{-\infty}^{\infty} \varpi(\sigma, x)[|x-\xi|+a(x-\xi)]^{p(1+\delta \sigma)-1} f^{p}(x) \mathrm{d} x\right\}^{\frac{1}{p}} .
$$

Hence, by (9), we derive (43).

By the reverse Hölder inequality (cf. [17]), we have

$$
I \geq J_{1}\left[\sum_{|n|=1}^{\infty}[|n-\eta|+b(n-\eta)]^{q(1-\sigma)-1} b_{n}^{q}\right]^{\frac{1}{q}} .
$$

Then by (43), we have (42).

On the other hand, assuming that (42) is valid, we set

$$
b_{n}:=[|n-\eta|+b(n-\eta)]^{p \sigma-1}\left[\int_{-\infty}^{\infty} H(x, n) f(x) \mathrm{d} x\right]^{p-1}(|n| \in \mathbf{N}) .
$$

Then we obtain that

$$
J_{1}=\left[\sum_{|n|=1}^{\infty}[|n-\eta|+b(n-\eta)]^{q(1-\sigma)-1} b_{n}^{q}\right]^{\frac{1}{p}} .
$$

In view of (45), it follows that $J_{1}>0$. If $J_{1}=\infty$, then (43) is trivially valid; if $J_{1}<\infty$, then by (42), we have

$$
\begin{aligned}
& \|b\|_{q, \Phi}^{q}=J_{1}^{p}=I>K_{a, b}(\sigma)\|f\|_{p, \Psi}\|b\|_{q, \Phi}, \\
& \|b\|_{q, \Phi}^{q-1}=J_{1}>K_{a, b}(\sigma)\|f\| \|_{p, \Psi},
\end{aligned}
$$

namely, (43) holds true, which is equivalent to (42).

Similarly, we have

$$
J_{2}>k_{b}^{\frac{1}{p}}(\sigma)\left\{\sum_{|n|=1}^{\infty} \omega(\sigma, n)[|n-\eta|+b(n-\eta)]^{q(1-\sigma)-1} b_{n}^{q}\right\}^{\frac{1}{q}} .
$$

Hence, by (8), we deduce (37). We have proved that (42) is satisfied. Setting

$$
f(x):=\frac{1}{[|x-\xi|+a(x-\xi)]^{q \delta \sigma+1}}\left(\sum_{|n|=1}^{\infty} H(x, n) b_{n}\right)^{q-1}(x \in \mathbf{R} \backslash\{\xi\}),
$$

it follows that

$$
J_{2}=\left\{\int_{-\infty}^{\infty}[|x-\xi|+a(x-\xi)]^{p(1+\delta \sigma)-1} f^{p}(x) \mathrm{d} x\right\}^{\frac{1}{q}},
$$

and in view of (47), we get $J_{2}>0$. If $J_{2}=\infty$, then (44) is trivially valid; if $J_{2}<\infty$, then by (42), we have 


$$
\begin{aligned}
& \|f\|_{p, \Psi}^{p}=J_{2}^{q}=I>K_{a, b}(\sigma)\|f\|_{p, \Psi}\|b\|_{q, \Phi}, \\
& \|f\|_{p, \Psi}^{p-1}=J_{2}>K_{a, b}(\sigma)\|b\|_{q, \Phi},
\end{aligned}
$$

namely, (44) follows.

On the other hand, assuming that (44) is valid, by the reverse Hölder inequality (cf. [17]), we obtain

$$
I \geq\left\{\int_{-\infty}^{\infty}[|x-\xi|+a(x-\xi)]^{p(1+\delta \sigma)-1} f^{p}(x) \mathrm{d} x\right\}^{\frac{1}{p}} J_{2} .
$$

Then by (44), we deduce (22), which is equivalent to (44). Therefore, inequalities (42), (43) and (44) are equivalent.

For $0<\varepsilon<|p| \sigma$, we set $\widetilde{\sigma}=\sigma+\frac{\varepsilon}{p}(>0)$,

$$
\tilde{f}(x):=\left\{\begin{array}{c}
\frac{1}{[|x-\xi|+a(x-\xi)]^{\delta\left(\sigma+\frac{\varepsilon}{p}\right)+1}}, \quad x \in E_{\delta}, \\
0, \quad x \in \mathbf{R} \backslash E_{\delta},
\end{array}\right.
$$

and

$$
\widetilde{b}_{n}:=[|n-\eta|+b(n-\eta)]^{\left(\sigma-\frac{\varepsilon}{q}\right)-1},|n| \in \mathbf{N} .
$$

Then by (12) and (14), we obtain that

$$
\begin{aligned}
\tilde{I}_{1}:= & \left\{\int_{-\infty}^{\infty}[|x-\xi|+a(x-\xi)]^{p(1+\delta \sigma)-1} \widetilde{f}^{p}(x) \mathrm{d} x\right\}^{\frac{1}{p}} \\
& \times\left\{\sum_{|n|=1}^{\infty}[|n-\eta|+b(n-\eta)]^{q(1-\sigma)-1} \widetilde{b}_{n}^{q}\right\}^{\frac{1}{q}} \\
= & \left\{\int_{-\infty}^{\infty} \frac{\mathrm{d} x}{[|x-\xi|+a(x-\xi)]^{\delta \varepsilon+1}}\right\}^{\frac{1}{p}}\left\{\sum_{|n|=1}^{\infty} \frac{1}{[|n-\eta|+b(n-\eta)]^{\varepsilon+1}}\right\}^{\frac{1}{q}} \\
= & \frac{1}{\varepsilon}\left(\frac{2}{1-a^{2}}\right)^{\frac{1}{p}}\left[\left(\frac{2}{1-b^{2}}+o_{1}(1)\right)\left(1+o_{2}(1)\right)^{\frac{1}{q}} .\right.
\end{aligned}
$$

By (9), we also have 


$$
\begin{aligned}
\widetilde{I} & :=\sum_{|n|=1}^{\infty} \int_{-\infty}^{\infty} H(x, n) \tilde{f}(x) \tilde{b}_{n} \mathrm{~d} x \\
& =\sum_{|n|=1}^{\infty} \int_{E_{\delta}} H(x, n) \frac{[|n-\eta|+b(n-\eta)]^{\left(\sigma-\frac{\varepsilon}{q}\right)-1}}{[|x-\xi|+a(x-\xi)]^{\delta\left(\sigma+\frac{\varepsilon}{p}\right)+1}} \mathrm{~d} x \\
& \leq \sum_{|n|=1}^{\infty} \int_{-\infty}^{\infty} H(x, n) \frac{[|n-\eta|+b(n-\eta)]^{(\widetilde{\sigma}-\varepsilon)-1}}{[|x-\xi|+a(x-\xi)]^{\delta \widetilde{\sigma}+1}} \mathrm{~d} x \\
& =\sum_{|n|=1}^{\infty} \frac{\omega(\widetilde{\sigma}, n)}{[|n-\eta|+b(n-\eta)]^{\varepsilon+1}} \\
& =k_{a}(\tilde{\sigma}) \sum_{|n|=1}^{\infty} \frac{1}{[|n-\eta|+b(n-\eta)]^{\varepsilon+1}} \\
& =\frac{1}{\varepsilon} k_{a}(\tilde{\sigma})\left(\frac{2}{1-b^{2}}+o_{1}(1)\right)\left(1+o_{2}(1)\right)
\end{aligned}
$$

If the constant factor $K_{a, b}(\sigma)$ in (42) is not the best possible, then there exists a positive number $k$, with $K_{a, b}(\sigma)<k$, such that (42) is satisfied when we replace $K_{a, b}(\sigma)$ by $k$. Then in particular, we have $\varepsilon \tilde{I}>\varepsilon k \widetilde{I}_{1}$, namely

$$
\begin{aligned}
& k_{a}\left(\sigma+\frac{\varepsilon}{p}\right)\left(\frac{2}{1-b^{2}}+o_{1}(1)\right)\left(1+o_{2}(1)\right) \\
& \quad>k \cdot\left(\frac{2}{1-a^{2}}\right)^{\frac{1}{p}}\left[\left(\frac{2}{1-b^{2}}+o_{1}(1)\right)\left(1+o_{2}(1)\right)\right]^{\frac{1}{q}}
\end{aligned}
$$

It follows that

$$
k_{a}(\sigma) \frac{2}{1-b^{2}} \geq k \cdot\left(\frac{2}{1-a^{2}}\right)^{1 / p}\left(\frac{2}{1-b^{2}}\right)^{1 / q}\left(\varepsilon \rightarrow 0^{+}\right),
$$

that is

$$
K_{a, b}(\sigma)=\frac{2\left[\frac{\pi}{\lambda \sin \left(\frac{\pi \sigma}{\lambda}\right)}\right]^{2}}{\left(1-a^{2}\right)^{1 / q}\left(1-b^{2}\right)^{1 / p}} \geq k .
$$

This is a contradiction. Hence, the constant factor $K_{a, b}(\sigma)$ in (42) is the best possible.

The constant factor $K_{a, b}(\sigma)$ in (43) ((44)) is still the best possible. Otherwise, we would reach a contradiction by (46) $((48))$ that the constant factor $K_{a, b}(\sigma)$ in (42) is not the best possible.

This completes the proof of the theorem.

Acknowledgements B. C. Yang: This work is supported by the National Natural Science Foundation (No. 61772140), and the Characteristic innovation project of Guangdong Provincial Colleges and universities in 2020 (No. 2020KTSCX088). We are grateful for their support. 
Funding Open Access funding provided by Universität Zürich.

Open Access This article is licensed under a Creative Commons Attribution 4.0 International License, which permits use, sharing, adaptation, distribution and reproduction in any medium or format, as long as you give appropriate credit to the original author(s) and the source, provide a link to the Creative Commons licence, and indicate if changes were made. The images or other third party material in this article are included in the article's Creative Commons licence, unless indicated otherwise in a credit line to the material. If material is not included in the article's Creative Commons licence and your intended use is not permitted by statutory regulation or exceeds the permitted use, you will need to obtain permission directly from the copyright holder. To view a copy of this licence, visit http://creativecommons.org/licen ses/by/4.0/.

\section{References}

1. Adiyasuren, V., Batbold, T., Krnić, M.: Half-discrete Hilbert-type inequalities with mean operators, the best constants, and applications. Appl. Math. Comput. 231, 148-159 (2014). https://doi. org/10.1016/j.amc.2014.01.011

2. Adiyasuren, V., Batbold, T., Krnić, M.: Multiple Hilbert-type inequalities involving some differential operators. Banach J. Math. Anal. 10(2), 320-337 (2016)

3. Basci, Y., Baleanu, D.: Hardy-type inequalities within fractional derivatives without singular kernel. J. Inequal. Appl. 2018, 304 (2018)

4. Batbold, T., Azar, L.E.: A new form of Hilbert integral inequality. J. Math. Inequal. 12(1), 379_ 390 (2018)

5. Brnetić, I., Krnić, M., Pečarić, J.: Multiple Hilbert and Hardy-Hilbert inequalities with non-conjugate parameters. Bull. Aust. Math. Soc. 71, 447-457 (2005)

6. Burtseva, E., Lundberg, S., Persson, L.E., Natasha, S.: Multi-dimensional Hardy type inequalities in Holder spaces. J. Math. Inequal. 12(3), 719-729 (2018)

7. Debnath, L., Yang, B. C.: Recent developments of Hilbert-type discrete and integral inequalities with applications. Int. J. Math. Math. Sci. Volume 2012, Article ID 871845, 29 pages

8. Fabelurin, O.O., Oguntuase, J.A., Persson, L.E.: Multidimensional Hardy-type inequality on time scales with variable exponents. J. Math. Inequal. 13(3), 725-736 (2019)

9. Gu, Z.H., Yang, B.C.: A Hilbert-type integral inequality in the whole plane with a non-homogeneous kernel and a few parameters. J. Inequal. Appl. 2015, 314 (2015)

10. Hardy, G. H.: Note on a theorem of Hilbert concerning series of positive terms. Proc. Lond. Math. Soc. 23(2), xlv-xlvi

11. Hardy, G.H., Littlewood, J. E., Pólya, G.: Inequalities. Cambridge University Press, Cambridge (1934)

12. He, B., Hong, Y., Li, Z., Yang, B.C.: Necessary and sufficient conditions and optimal constant factors for the validity of multiple integral half-discrete Hilbert type inequalities with a class of quasi-homogeneous kernels. J. Appl. Anal. Comput. 11(1), 521-531 (2021)

13. He, B., Yang, B.C.: On a Hilbert-type integral inequality with the homogeneous kernel of 0-degree and the hypergeometrc function. Math. Pract. Theory 40(18), 203-211 (2010)

14. Huang, Q.L., Wu, S.H., Yang, B.C.: Parameterized Hilbert-type integral inequalities in the whole plane. Sci. World J., Volume 2014, Article ID 169061, 8 pages

15. Jaksetic, J., Pecarc, J., Kalamir, K.S.: Further improvement of an extension of Holder-type inequality. Math. Inequal. Appl. 22(4), 1161-1175 (2019)

16. Krnić, M., Vuković, P.: Multidimensional Hilbert-type inequalities obtained via local fractional calculus. Acta Appl. Math. 169(1), 667-680 (2020). https://doi.org/10.1007/s10440-020-00317-x

17. Kuang, J.C.: Applied Inequalities. Shangdong Science and Technology Press, Jinan (2004)

18. Kuang, J.C.: Real and Functional Analysis, vol. 2. Higher Education Press, Beijing (2015)

19. Liu, Q.: A Hilbert-type integral inequality under configuring free power and its applications. J. Inequal. Appl. 2019, 91 (2019)

20. Milovanovic, G.V., Rassias, M.T.: Some properties of a hypergeometric function which appear in an approximation problem. J. Glob. Optim. 57, 1173-1192 (2013) 
21. Mitrinović, D. S., Pecaric, J. E., Fink, A. M.: Inequalities Involving Functions and their Integrals and Derivatives. Kluwer Academic, Boston (1991)

22. Rassias, M.T., Yang, B.C.: On half-discrete Hilbert's inequality. Appl. Math. Comput. 220, 75-93 (2013)

23. Rassias, M.T., Yang, B.C.: A multidimensional half - discrete Hilbert - type inequality and the Riemann zeta function. Appl. Math. Comput. 225, 263-277 (2013)

24. Rassias, M.T., Yang, B.C.: On a multidimensional half - discrete Hilbert - type inequality related to the hyperbolic cotangent function. Appl. Math. Comput. 42, 800-813 (2013)

25. Rassias, M.T., Yang, B.C.: A Hilbert - type integral inequality in the whole plane related to the hyper geometric function and the beta function. J. Math. Anal. Appl. 428(2), 1286-1308 (2015)

26. Rassias, M.T., Yang, B.C.: Equivalent properties of a Hilbert-type integral inequality with the best constant factor related to the Hurwitz zeta function. Ann. Funct. Anal. 9(2), 282-295 (2018)

27. Wang, A.Z., Yang, B.C.: A new Hilbert-type integral inequality in whole plane with the nonhomogeneous kernel. J. Inequal. Appl. 2011, 123 (2011)

28. Xin, D.M.: A Hilbert-type integral inequality with the homogeneous kernel of zero degree. Math. Theory Appl. 30(2), 70-74 (2010)

29. Xin, D.M., Yang, B.C., Chen, Q.: A discrete Hilbert-type inequality in the whole plane. J. Inequal. Appl. 2016, 133 (2016)

30. Xin, D. M., Yang, B. C.: A Hilbert-type integral inequality in whole plane with the homogeneous kernel of degree -2. J Inequal. Appl. Vol. 2011, Article ID 401428, 11 pages

31. Xu, J.S.: Hardy-Hilbert's inequalities with two parameters. Adv. Math. 36(2), 63-76 (2007)

32. Yang, B.C.: On the norm of an integral operator and applications. J. Math. Anal. Appl. 321, 182$192(2006)$

33. Yang, B.C.: A new Hilbert-type integral inequality. Soochow J. Math. 33(4), 849-859 (2007)

34. Yang, B.C.: A new Hilbert-type integral inequality with some parameters. J. Jilin Univ. (Sci. Ed.) 46(6), 1085-1090 (2008)

35. Yang, B.C.: A Hilbert-type integral inequality with a non-homogeneous kernel. J. Xiamen Univ. (Nat. Sci.) 48(2), 165-169 (2008)

36. Yang, B.C.: The Norm of Operator and Hilbert-Type Inequalities. Science Press, Beijing (2009)

37. Yang, B.C.: A survey of the study of Hilbert-type inequalities with parameters. Adv. Math. 38(3), 257-268 (2009)

38. Yang, B.C.: A Hilbert-type integral inequality with the homogenous kernel of degree 0. J. Shandong Univ. (Nat. Sci.) 45(2), 103-106 (2010)

39. Yang, B.C.: A half-discrete Hilbert's inequality. J. Guangdong Univ. Educ. 31(3), 1-7 (2011)

40. Yang, B.C., Debnath, L.: Half-Discrete Hilbert-Type Inequalitiea. World Scientific Publishing, Singapore (2014)

41. Yang, B., Krnić, M.: A half-discrete version of the Mulholland inequality. Math. Rep. 66(2), 163174 (2014)

42. You, M.H., Guan, Y.: On a Hilbert-type integral inequality with non-homogeneous kernel of mixed hyperbolic functions. J. Math. Inequal. 13(4), 1197-1208 (2019)

43. Zeng, Z., Z. Xie, Z. T.: On a new Hilbert-type integral inequality with the homogeneous kernel of degree 0 and the integral in whole plane. J. Inequal. Appl. Vol. 2010, Article ID 256796, 9 pages

44. Zeng Z.Z., Raja Rama Gandhi, K., Xie, Z. T.: A new Hilbert-type inequality with the homogeneous kernel of degree -2 and with the integral. Bull. Math. Sci. Appl. 3(1), 11-20 (2014)

45. Zhao, C.J., Cheung, W.S.: On Hilberts inequalities with alternating signs. J. Math. Inequal. 12(1), 191-200 (2018)

46. Zhao, C.J., Cheung, W.S.: Reverse Hilbert type inequalities. J. Math. Inequal. 13(3), 855-866 (2019)

47. Zhong, J.H., Chen, Q.: A half-discrete Hilbert-type inequality with the decreasing and homogeneous kernel of degree 0 (Chinese). J. Zhejiang Univ. Sci. Ed. 42(1), 77-81 (2015) 